Open Access

\title{
Substantial reprogramming of the Eutrema salsugineum (Thellungiella salsuginea) transcriptome in response to UV and silver nitrate challenge
}

\author{
Stefanie Mucha', Dirk Walther², Teresa M Müller', Dirk K Hincha² and Erich Glawischnig ${ }^{1 *}$
}

\begin{abstract}
Background: Cruciferous plants synthesize a large variety of tryptophan-derived phytoalexins in response to pathogen infection, UV irradiation, or high dosages of heavy metals. The major phytoalexins of Eutrema salsugineum (Thellungiella salsuginea), which has recently been established as an extremophile model plant, are probably derivatives of indole glucosinolates, in contrast to Arabidopsis, which synthesizes characteristic camalexin from the glucosinolate precursor indole-3-acetaldoxime.

Results: The transcriptional response of E. salsugineum to UV irradiation and $\mathrm{AgNO}_{3}$ was monitored by RNAseq and microarray analysis. Most transcripts (respectively $70 \%$ and $78 \%$ ) were significantly differentially regulated and a large overlap between the two treatments was observed (54\% of total). While core genes of the biosynthesis of aliphatic glucosinolates were repressed, tryptophan and indole glucosinolate biosynthetic genes, as well as defence-related WRKY transcription factors, were consistently upregulated. The putative Eutrema WRKY33 ortholog was functionally tested and shown to complement camalexin deficiency in Atwrky33 mutant.
\end{abstract}

Conclusions: In E. salsugineum, UV irradiation or heavy metal application resulted in substantial transcriptional reprogramming. Consistently induced genes of indole glucosinolate biosynthesis and modification will serve as candidate genes for the biosynthesis of Eutrema-specific phytoalexins.

Keywords: Eutrema salsugineum, Thellungiella salsuginea, Transcriptomics, Glucosinolate biosynthesis, Phytoalexin

\section{Background}

The synthesis of bioactive compounds for adaptation to abiotic stress conditions and for defence against herbivores and pathogen infections is a fundamental survival strategy of plants. The biosynthesis of phytoalexins, which contain an indole moiety substituted with additional ring systems or side chains, often containing sulphur and nitrogen, is characteristic for cruciferous plants [1]. The individual structures are very diverse even among different Brassica cultivars. In Arabidopsis thaliana, a variety of compounds are synthesized from the intermediate indole-3-acetonitrile (IAN) in response

\footnotetext{
* Correspondence: egl@wzw.tum.de

${ }^{1}$ Lehrstuhl für Genetik, Technische Universität München, D-85354 Freising, Germany

Full list of author information is available at the end of the article
}

to pathogen infection or heavy metal stress $[2,3]$ with camalexin as the most prominent metabolite. The camalexin biosynthetic pathway from tryptophan and glutathione and its role in defence against a number of fungal pathogens has been investigated in detail [4]. Phytoalexin biosynthesis is induced upon pathogen infection, but also under harsh abiotic conditions, such as high dosages of heavy metal ions or UV light, which lead to the generation of reactive oxygen species and ultimately to programmed cell death. For studies on plant metabolism, abiotic stress treatments provide the advantage that no interference of pathogen metabolism, which is often strain specific [5], has to be taken into account.

Eutrema salsugineum has been established recently as an alternative model system for crucifers in addition to Arabidopsis, because of its high tolerance of various 
abiotic stresses [6]. The E. salsugineum genome sequence $[7,8]$, as well as a reference transcriptome, [9] are available and additional transcriptomics data were published recently $[8,10]$. E. salsugineum is also referred to as Thellungiella salsuginea. The ecotype Shandong analysed in this study was initially assigned as T. halophila and this species name was used in a number of publications [11-13]. Consequently, gene and transcript sequences isolated from Shandong ecotype have been deposited under the species names T. halophila, T. salsuginea and E. salsugineum. According to work by Koch and German [14], the species name T. salsuginea is acceptable, but E. salsugineum, which we refer to in this manuscript, is preferred.

Within the Brassicaceae, Eutrema and Arabidopsis are rather distantly related and their last common ancestor is estimated to have lived 43 million years ago [8]. Still, large stretches of syntenic regions were identified in the genomes, allowing clear assignment of putative orthologs $[7,8]$. At the protein level, for the number of best hit pairs between Eutrema and Arabidopsis a peak at 85\% amino acid sequence identity was determined [8].

Eutrema and Arabidopsis have developed a diversified spectrum of defence compounds, such as glucosinolates $[11,15,16]$ and indolic phytoalexins. In Arabidopsis, these phytoalexins are predominantly synthesized from the intermediate indole-3-acetaldoxime [2,17], while the characteristic Eutrema phytoalexins are most likely derivatives of 1-methoxy-indole glucosinolate [18]. The identification of biosynthetic genes for presumably glucosinolatederived (Eutrema) and glucosinolate-independent (Arabidopsis) phytoalexins will build the basis for metabolic engineering studies of indolic phytoalexins and for establishment of a model for phytoalexin evolution in the Brassicaceae.

In this work, we analysed the transcriptional reprogramming of E. salsugineum in response to abiotic stress conditions, which lead to the accumulation of phytoalexins. We show that genes of tryptophan and indole glucosinolate biosynthesis and modification are highly upregulated providing candidates for phytoalexin biosynthesis. Also the Eutrema ortholog of WRKY33, a key regulator of Arabidopsis phytoalexin induction, was highly upregulated, even though known WRKY33 target genes, such as CYP71B15 [19] are apparently missing in E. salsugineum.

\section{Results and Discussion}

Induction of phytoalexin biosynthesis in response to UV light and silver nitrate spraying

The biosynthesis of phytoalexins by Brassicaceae species is induced by pathogen infection, but also specific abiotic stress treatments, such as high dosages of heavy metals and UV light. Applying abiotic stressors provides the advantage of a high degree of experimental reproducibility and excludes the modulation of plant defence reactions and metabolism by the pathogen. Induction of phytoalexin biosynthesis by the heavy metal salt $\mathrm{CuCl}$ and UV treatment was previously established by Pedras and coworkers [12,13]. Here, wasalexin induction was confirmed for 10-week old E. salsugineum (Shandong) leaves in response to UVC light, silver nitrate application, and Botrytis cinerea infection (Additional file 1: Figure S1).

In Arabidopsis, expression of camalexin biosynthetic genes is coregulated with expression of $A S A 1$, encoding the committing enzyme of tryptophan biosynthesis. We therefore assumed that also in E. salsugineum tryptophan biosynthesis is upregulated under phytoalexin inducing conditions, which we later confirmed (see below). Quantitative RT-PCR was used to determine the induction kinetics of EsASA1 (Figure 1). For both treatments, transcript levels were highly elevated $7.5 \mathrm{~h}$ and $10 \mathrm{~h}$ after the onset of induction. Therefore, for transcriptomics analysis $8 \mathrm{~h}$ induction was selected.

\section{The Eutrema transcriptome in response to UV light and heavy metal stress}

RNA was isolated from non-treated leaves and from leaves treated with either $\mathrm{AgNO}_{3}$ or UV light. cDNA libraries were prepared and approximately 33 Mio to 45 Mio $50 \mathrm{bp}$ reads per library were obtained by Illumina sequencing. Reads were mapped to the JGI genome [8]. For each cDNA library, approx. $75 \%$ of total transcript models were covered (Table 1) and a large overlap between treatments was observed (Additional file 2: Figure S2). Transcript models were analysed for read-counts in the different samples and annotated for best hit in the Arabidopsis thaliana genome (Additional file 3: Table S1).

Similarly, we have analysed the transcriptome $48 \mathrm{~h}$ after infection of plants with $B$. cinerea (Additional file 3: Table S1). 3139 transcripts were identified as more than 2-fold upregulated with respect to untreated leaves. Of this set, $56 \%$ and $61 \%$ were also upregulated more than 2-fold after $\mathrm{UV}$ and $\mathrm{AgNO}_{3}$ treatment, respectively, indicating overlapping responses to the abiotic and biotic stressors. However, as transcriptional changes in response to UV light and $\mathrm{AgNO}_{3}$ were much more pronounced, we focussed on these treatments for further analysis.

Microarray analysis of four biological replicates was conducted with Agilent arrays based on the design by Lee et al. [9]. Statistically robust differential regulation was observed for the majority of transcripts (Additional file 4: Table S2). Of a total of 42562 oligonucleotide probes, signal intensities of 11930 (28\%) and 15384 (36\%) probes were significantly (t-test FDR corrected $\mathrm{p}<0.01$ ) elevated, while signal intensities of 11562 (27\%) and 11879 (28\%) 

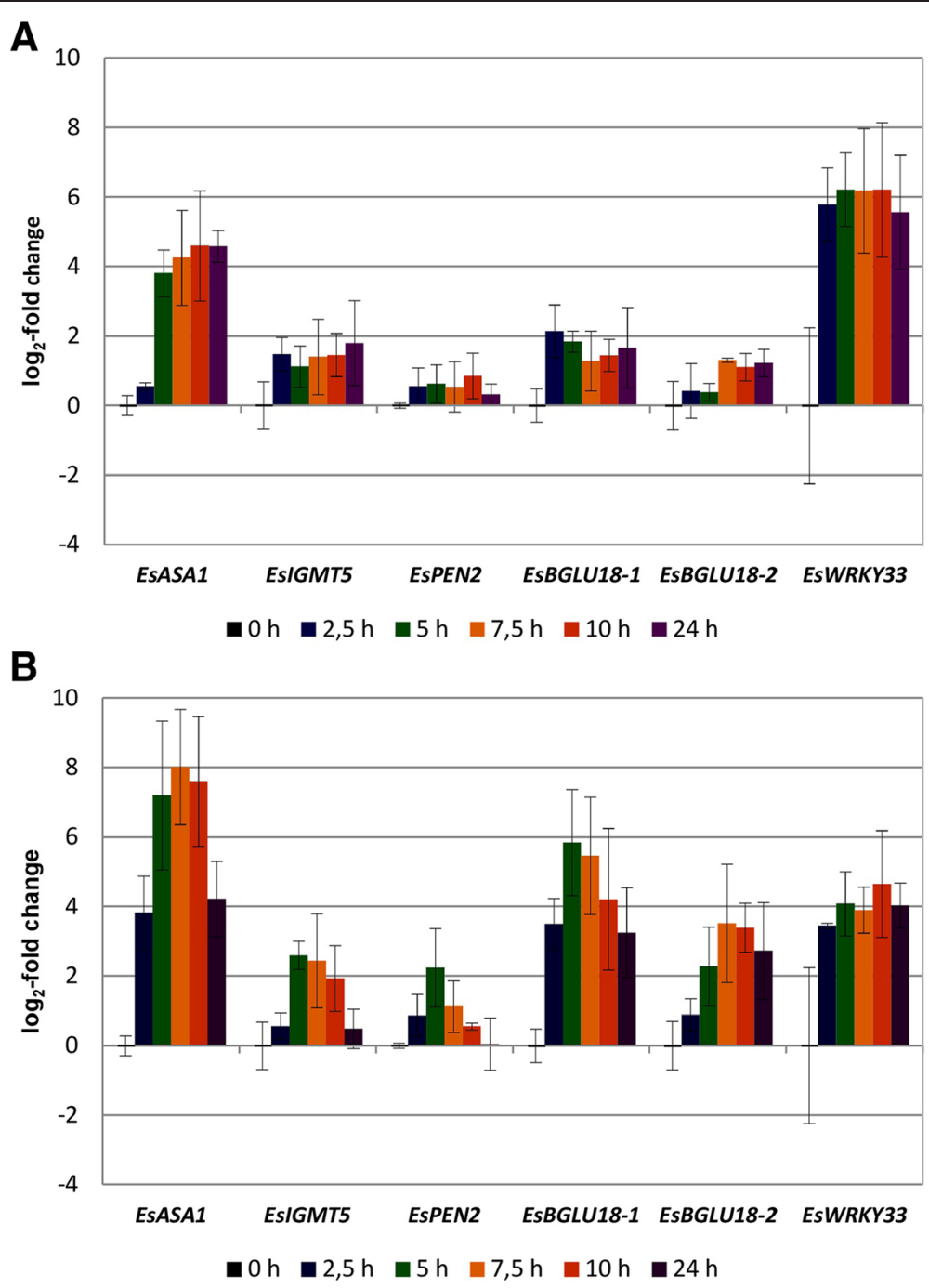

Figure 1 RT-qPCR analysis. Time course of expression after treatment with UV light (A) and $\mathrm{AgNO}_{3}$ (B). EsASA1 (Thhalv10013041m), Es/GMT5 (Thhalv10018739m), EsPEN2 (Thhalv10001354m), EsBGLU18-1 (Thhalv10011384m), EsBGLU18-2 (Thhalv10011385m), and EsWRKY33 (Thhalv10016542m), were analysed. The expression levels, relative to the mean for $0 \mathrm{~h}$, were determined by RT-qPCR, normalized to the geometric mean of three reference genes (EsActin1, ESYLS8 and ESPP2AA2). Values are means of three independent experiments \pm SE.

Table 1 RNAseq metrics and alignments

\begin{tabular}{llllll}
\hline & & n.i. & UV & AgNO $_{\mathbf{3}}$ & B.c. \\
\hline reads & total fragments & $33,445,682$ & $45,326,703$ & $33,278,110$ & $35,924,995$ \\
& uncounted & $8,100,893$ & $22,525,573$ & $7,470,863$ & $12,091,407$ \\
& counted & $25,344,789$ & $22,801,130$ & $25,807,247$ & $19,065,875$ \\
& - uniquely & $17,567,426$ & $14,322,990$ & $6,741,372$ & $16,287,764$ \\
transcripts & - non-specific & $7,777,363$ & $8,478,140$ & 23,985 & $2,545,824$ \\
& hit (reads $>0)$ & 23,237 & 23,730 & 22,216 & 22,655 \\
& uniquely hit & 21,589 & 21,875 & $(75,9 \%)$ & $(75,3 \%)$ \\
\hline
\end{tabular}

Reads were mapped to the JGI genome (Yang et al., [8]), 29284 reference transcripts (2 mismatches allowed); uncounted/counted: number of unmapped/mapped reads; uniquely: number of uniquely mapped reads; non-specific: number of reads with multiple locations in the reference. 
probes were significantly reduced in response to UV light and $\mathrm{AgNO}_{3}$, respectively.

These array data were compared with the RNAseq data, which in addition provide information about absolute expression levels. A correlation analysis with the $\log _{2}$ fold-change values obtained by the two methods in response to UV and $\mathrm{AgNO}_{3}$ is shown in Additional file 5: Figure S3.

We matched RNAseq and array data based on the comparison of array probe and transcript model sequences and omitted those probes from further analysis for which no match was found. Duplicated genes with highly homologous sequences were sometimes indistinguishable on array level (e.g. TsCYP79B2, see below). Here, the more highly abundant transcript from the RNAseq analysis was chosen for the matched dataset. $\log _{2}$ fold-change values based on RNAseq and array analyses were correlated $(r=0.66$ for UV light, $r=0.65$ for $\mathrm{AgNO}_{3}$ ). For further analysis, we worked with a set of 14,706 genes, for which both array and RNAseq data are available (Additional file 6: Table S3). Correlations of $\log _{2}$ fold-change values in response to $\mathrm{UV}$ and $\mathrm{AgNO}_{3}$ treatment obtained by microarray hybridization are shown in Figure 2. For a large proportion of these transcripts (88\%), significant changes in abundance were detected in response to $\mathrm{UV}$ or $\mathrm{AgNO}_{3}$ treatment (Figure 2). 4502 (31\%) transcripts were upregulated, 3433 (23\%) downregulated in response to both treatments, indicating substantial overlap in metabolic and regulatory responses.

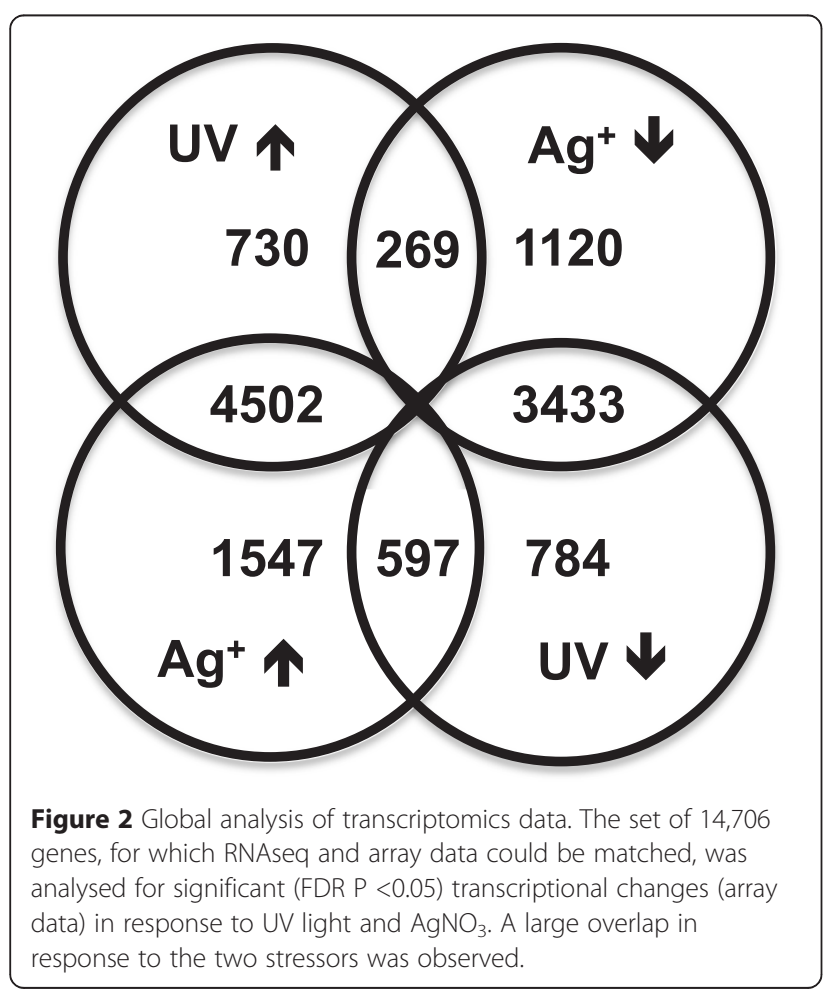

Figure 3 shows a Mapman [20] representation of $\log _{2}-$ fold transcriptional changes, in response to UV light (Figure 3A) and $\mathrm{AgNO}_{3}$ (Figure 3B), based on array data. Strongly repressed processes include photosynthesis and starch synthesis. The tricarboxylic acid cycle, providing precursors of aromatic amino acid and the biosynthesis of cell wall precursors are induced on the level of transcript abundance, consistent with plant defence reactions.

\section{Transcriptional changes induced upon both UV and heavy metal stress}

Transcripts that were strongly and consistently upregulated in response to both UV light and $\mathrm{AgNO}_{3}$ include a number of genes that encode enzymes involved in biosynthesis or modification of hormones and signalling compounds. This indicated that reprogramming the hormone balance is one of the key elements in the adaptation of Eutrema to high dosages of UV light or heavy metals. Genes upregulated most strongly in response to both stressors include EsSOT12 and, based on NGS data, EsST2a/EsSOT1 (Additional file 3: Table S1 and Additional file 6: Table S3). The corresponding Arabidopsis orthologs encode a sulfotransferase, which sulphonates salicylic acid, thereby positively regulating salicylic acid accumulation [21], and a sulfotransferase, which sulphonates hydroxyjasmonic acid [22]. SOT12 is also strongly induced in $A$. thaliana seedlings in response to UVB light [23]. Furthermore, we observed that genes encoding Eutrema orthologs of 1-amino-cyclopropane-1carboxylate synthase 2 (ethylene biosynthesis) and ciszeatin O- $\beta$-D-glucosyltransferase (UGT85A1, cytokinin metabolism) [24] were highly upregulated in response to both UV light and $\mathrm{AgNO}_{3}$. Other induced processes are senescence and regulation of cell death. Here, examples of highly upregulated genes include the Eutrema orthologs of AtDLAH [25] and AtBAP2, an inhibitor of programmed cell death [26].

We observed significant transcriptional reprogramming of phenylpropanoid metabolism. Genes of the core phenylpropanoid biosynthetic pathway, i.e. E. salsugineum orthologs putatively encoding phenylalanine ammonia-lyase 1 and 2, cinnamate-4-hydroxylase, cinnamoyl CoA reductase, and cinnamyl alcohol dehydrogenase were upregulated in response to $\mathrm{UV}$ and $\mathrm{AgNO}_{3}$. The E. salsugineum ortholog of TT4, encoding naringenine chalcone synthase, was strongly downregulated. Interestingly, in Arabidopsis strong TT4 upregulation was observed in response to UV light [27]. Whether this is due to experimental differences, such as plant age or UV wavelength or reflects a species-specific difference in adaptation with respect to the phenylpropanoids that are synthesized remains to be investigated. Further, fundamental changes in the transcript abundance of genes encoding enzymes involved in the biosynthesis of defence- 


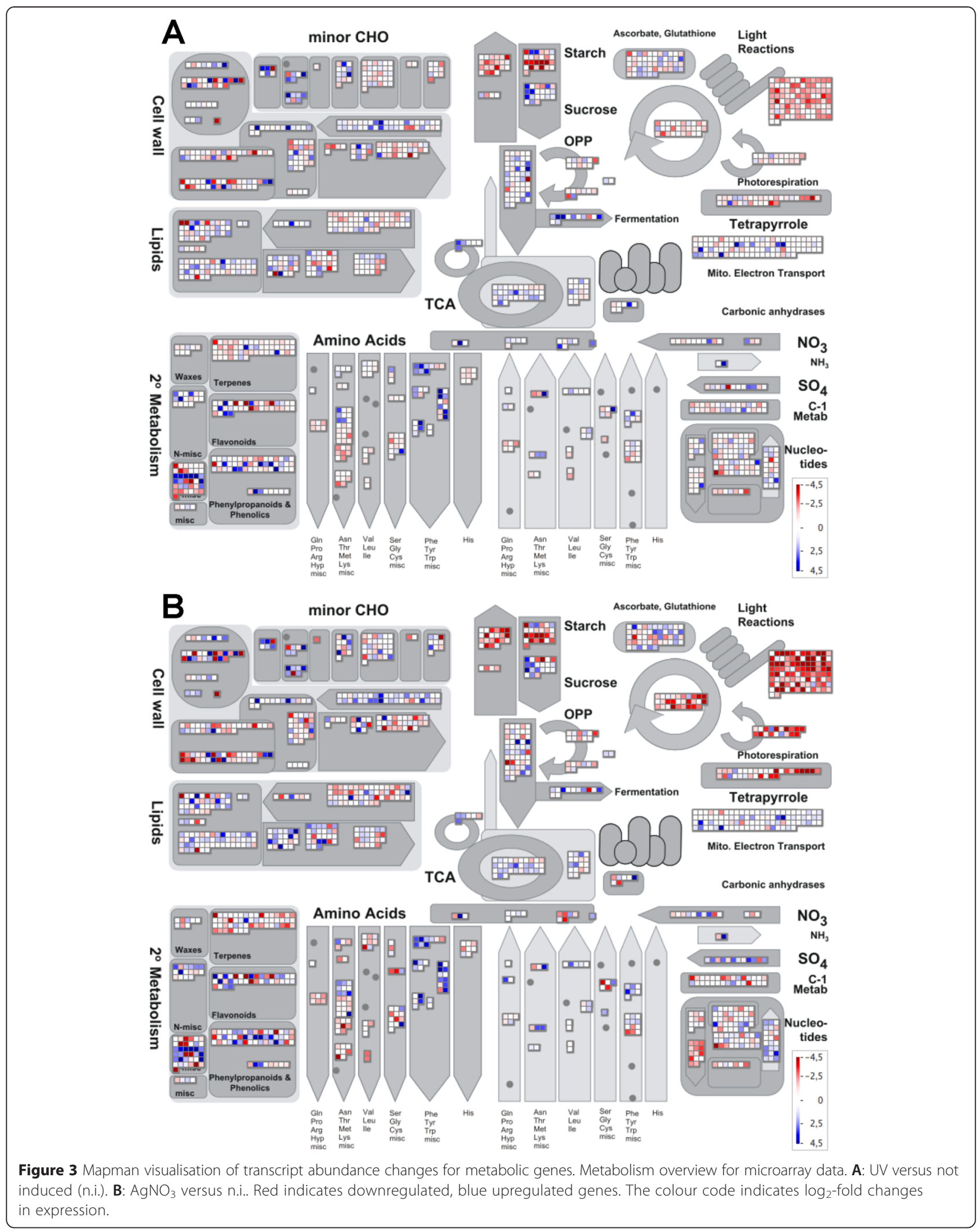


related secondary metabolites were observed, which are discussed in detail below.

A number of genes have been functionally associated with the halophytic lifestyle of E. salsugineum. These include the sodium transporter EsHKT1 [28] and EsERF1 [29], which are also strongly and significantly upregulated under both $\mathrm{AgNO}_{3}$ and $\mathrm{UV}$ treatment (Additional file 6: Table S3). Arabidopsis ERF1 is an integrator of different abiotic and biotic stress responses [30]. For other genes associated with salt tolerance, such as SOS1 and iron superoxide dismutase this was not observed [31]. We have surveyed transcriptional changes in response to $\mathrm{AgNO}_{3}$ and UV in E. salsugineum for similarity to changes in response to drought or cold [32]. There was a clear overlap among downregulated genes, which are mainly related to photosynthesis. A common pattern among upregulated genes was not observed (Additional file 7: Figure S4A). Apparently, the responses of E. salsugineum to drought/cold and to UV/heavy metal stresses differ substantially.

The effect of silver treatment on the Arabidopsis transcriptome was investigated previously by Kaveh and coworkers [33]. The number of significantly upregulated genes was much lower than in our work on Eutrema, probably due to differences in the experimental setup. Only for a few genes, the corresponding orthologs were identified in both studies, including the orthologs of the $\beta$-glucosidase genes 18 and 46 .

Recently, genes were identified in A. thaliana which are upregulated in response to both $B$. cinerea infection and oxidative stress [34]. For 115 out of these 175 transcripts, corresponding E. salsuginea orthologs were identified here. Strikingly, for a large fraction of these genes (76; 66\%), including e.g. EsCYP79B3 and EsCYP83B1 (see below), we observed upregulation by both UV and $\mathrm{AgNO}_{3}$ treatments (Additional file 7: Figure S4B). Possibly, all these processes lead to the generation of reactive oxygen species, inducing transcriptional reactions that are largely conserved between Arabidopsis and Eutrema.

\section{Tryptophan biosynthetic genes}

In Brassicaceae, tryptophan is a precursor of indole glucosinolates and indolic phytoalexins [4], which constitute the major tryptophan sinks. As cellular tryptophan concentrations are low in Arabidopsis leaves, tryptophan biosynthesis is strongly coregulated with the biosynthesis of camalexin $[35,36]$.

Here, we observed significant and strong increases in transcript levels associated with the tryptophan biosynthetic pathway in response to UV light and $\mathrm{AgNO}_{3}$ (Table 2). This includes genes encoding tryptophan synthase $\beta$ (TSB) type 1 isoforms, while the ortholog of TSBtype2, of which the biological function is unknown [37], is significantly downregulated in response to UV light.

\section{Glucosinolate biosynthesis and modification}

Members of the order Brassicales synthesize glucosinolates from non-polar amino acids as major defence compounds against herbivores and pathogens. In Arabidopsis thaliana, almost exclusively methionine-derived aliphatic and tryptophan-derived indole glucosinolates are found. Their biosynthetic pathways are known in great detail [38]. In Eutrema salsugineum Shandong, the short chain aliphatic allyl-2-phenylethyl-, 3-methylsulfinylpropyl-, and 3-methylthiopropylglucosinolate, the very-long-chain aliphatic 10-methylsulfinyldecylglucosinolate, as well as 3-indolylmethyl- and 1-methoxy-3-indolylmethylglucosinolate were identified as major compounds [11] (E. salsugineum denoted in this publication as T. halophila). According to labelling experiments, 1-methoxy-3-indolylmethylglucosinolate is likely to be a biosynthetic intermediate of the phytoalexins 1-methoxybrassinin and wasalexin A and B [18].

For all defined steps of the core aliphatic and indole glucosinolate biosynthetic pathways, putative orthologs of the genes encoding the corresponding enzymes were found in Eutrema salsugineum, based on homology and synteny to $A$. thaliana. Some additional duplication events or losses of tandem copies were detected. In contrast to the tandem duplicates CYP79F1 and CYP79F2 in A. thaliana, only one copy, designated as EsCYP79F1 was found in E. salsugineum, suggesting that this single gene is essential for the biosynthesis of aliphatic glucosinolates. A putative CYP79A2 [39] ortholog was found, which is expressed at very low levels $(0,0$, and 1 reads in n.i., UV, and $\mathrm{AgNO}_{3}$ samples, respectively) consistent with the apparent absence of phenylalanine-derived glucosinolates [11]. E. salsugineum contains three CYP79B genes due to a recent duplication of $C Y P 79 B 2$ leading to two transcripts hybridizing to the same array probe and generating proteins with $98.6 \%$ identity of their amino acid sequences. These two duplicates strongly differ in expression level based on RNAseq data (254, 24763 and 30609, versus 0, 3, and 5 reads in n.i., UV, and $\mathrm{AgNO}_{3}$ samples, respectively).

In response to $\mathrm{UV}$ light and $\mathrm{AgNO}_{3}$, the core genes of indole glucosinolate biosynthesis are strongly upregulated, consistent with the proposed role of 1-methoxy-3-indolylmethylglucosinolate as precursor of the characteristic Eutrema phytoalexins (Table 2). Also, the ortholog of MYB51/HIG1, encoding a master regulator of indole glucosinolate biosynthesis in Arabidopsis [40], is consistently induced. Strikingly, in response to these stressors, transcripts encoding indole glucosinolate biosynthetic genes, such as EsCYP83B1 and EsGGP1 are among the most highly abundant, according to our RNAseq data, indicating an important metabolic response.

In Arabidopsis, a time course experiment has been performed for UV response [41]. We surveyed these data 
Table 2 Analysis of transcript abundance changes of genes associated with the biosynthesis of defence-related metabolites

\begin{tabular}{|c|c|c|c|c|c|c|c|c|c|c|c|c|}
\hline \multirow[t]{2}{*}{ Transcript ID } & \multirow[t]{2}{*}{ Best Ath hit } & \multirow[t]{2}{*}{ Gene symbol } & \multirow[t]{2}{*}{ Annotation } & \multirow[t]{2}{*}{ UV } & \multirow[t]{2}{*}{$\mathrm{Ag}^{+}$} & \multirow{2}{*}{$\begin{array}{l}\text { Fold } \\
\text { change } \\
\text { log2 } \\
\text { (UV/n.i.) }\end{array}$} & \multirow{2}{*}{$\begin{array}{l}\text { FDR-p-value } \\
\text { test }\end{array}$} & \multirow{2}{*}{$\begin{array}{l}\text { Fold } \\
\text { change } \\
\text { log2 } \\
\text { (Ag/n.i.) }\end{array}$} & \multirow{2}{*}{$\begin{array}{l}\text { FDR-p-value } \\
\text { test }\end{array}$} & \multicolumn{3}{|c|}{ RNAseq Unique reads } \\
\hline & & & & & & & & & & n.i. & UV & AgNO3 \\
\hline \multicolumn{13}{|c|}{ Tryptophan biosynthesis } \\
\hline Thhalv10013041m & AT5G05730.1 & ASA1,TRP5,WEI2 & anthranilate synthase alpha subunit 1 & up & up & 4,83 & 0,000 & 5,31 & 0,000 & 431 & 20815 & 28637 \\
\hline Thhalv10010558m & AT3G54640.1 & TRP3,TSA1 & tryptophan synthase alpha chain & up & up & 4,34 & 0,000 & 3,76 & 0,000 & 264 & 9972 & 9692 \\
\hline Thhalv10013439m & AT4G27070.1 & TSB2 & tryptophan synthase beta-subunit 2 & up & up & 4,07 & 0,000 & 3,13 & 0,000 & 710 & 3153 & 2355 \\
\hline Thhalv10025097m & AT4G27070.1 & TSB2 & tryptophan synthase beta-subunit 2 & up & up & 3,37 & 0,000 & 2,47 & 0,000 & 2187 & 7481 & 5464 \\
\hline Thhalv10013857m & AT5G17990.1 & PAT1,TRP1 & tryptophan biosynthesis 1 & up & up & 2,26 & 0,000 & 3,26 & 0,000 & 22 & 207 & 268 \\
\hline Thhalv10014630m & AT4G27070.1 & TSB2 & tryptophan synthase beta-subunit 2 & up & up & 1,67 & 0,002 & 1,11 & 0,002 & 2 & 35 & 17 \\
\hline Thhalv10002557m & AT2G04400.1 & IGPS & indole-3-glycerol phosphate synthase & up & up & 1,16 & 0,000 & 1,40 & 0,000 & 684 & 6529 & 8538 \\
\hline Thhalv10016377m & AT2G29690.1 & ASA2 & anthranilate synthase 2 & & down & $-0,20$ & 0,356 & $-0,43$ & 0,004 & 482 & 436 & 449 \\
\hline Thhalv10027732m & AT5G38530.1 & TSBtype2 & tryptophan synthase beta type 2 & down & & $-1,47$ & 0,000 & $-1,40$ & 0,000 & 876 & 407 & 1012 \\
\hline \multicolumn{13}{|c|}{ Biosynthesis of aliphatic glucosinolates } \\
\hline Thhalv10023453m & AT1G62570.1 & FMO GS-OX4 & glucosinolate S-oxygenase 4 & up & up & 4,12 & 0,001 & 3,92 & 0,001 & 243 & 8147 & 7570 \\
\hline Thhalv10007582m & AT1G12140.1 & FMO GS-OX5 & glucosinolate S-oxygenase 5 & up & up & 2,12 & 0,000 & 1,48 & 0,000 & 604 & 1102 & 1077 \\
\hline Thhalv10018813m & AT1G74090.1 & ATST5B,SOT18 & desulfo-glucosinolate sulfotransf. 18 & & & 2,12 & 0,000 & 1,75 & 0,000 & 1133 & 461 & 175 \\
\hline Thhalv10007073m & AT1G18500.1 & IPMS1,MAML-4 & methylthioalkylmalate synthase-like 4 & & & 0,31 & 0,084 & 0,32 & 0,165 & 1695 & 1815 & 2672 \\
\hline Thhalv10004037m & AT5G23010.1 & IMS3,MAM1 & methylthioalkylmalate synthase 1 & & down & $-0,60$ & 0,055 & $-2,92$ & 0,000 & 51 & 1 & 4 \\
\hline Thhalv10017125m & AT2G43100.1 & ATLEUD1,IPMI2 & isopropylmalate isomerase 2 & down & & $-0,97$ & 0,002 & $-1,22$ & 0,003 & 655 & 462 & 942 \\
\hline Thhalv10013695m & AT5G14200.1 & IMD1 & isopropylmalate dehydrogenase 1 & down & down & $-1,97$ & 0,000 & $-1,91$ & 0,000 & 1667 & 266 & 779 \\
\hline Thhalv10028851m & AT4G12030.2 & BASS5,BAT5 & bile acid transporter 5 & down & down & $-2,05$ & 0,007 & $-4,56$ & 0,000 & 11 & 3 & 2 \\
\hline Thhalv10024982m & AT4G13770.1 & CYP83A1,REF2 & cytochrome P450 83A1 & down & down & $-2,79$ & 0,000 & $-3,97$ & 0,000 & 20919 & 1569 & 2903 \\
\hline Thhalv10007301m & AT1G16410.1 & CYP79F1 & cytochrome P450 79 F1 & down & down & $-2,92$ & 0,005 & $-5,72$ & 0,000 & 15712 & 1101 & 2740 \\
\hline Thhalv10013952m & AT5G07690.1 & MYB29 & myb domain protein 29 & down & down & $-3,22$ & 0,002 & $-4,35$ & 0,000 & 3421 & 380 & 61 \\
\hline Thhalv10004406m & AT5G61420.2 & MYB28,HAG1 & myb domain protein 28 & down & down & $-5,60$ & 0,000 & $-6,10$ & 0,001 & 427 & 10 & 10 \\
\hline \multicolumn{13}{|c|}{ Indole and general glucosinolate biosynthesis } \\
\hline Thhalv10007957m & AT1G21100.1 & IGMT1 & O-methyltransferase family protein & up & up & 5,97 & 0,000 & 4,54 & 0,001 & 165 & 10234 & 6500 \\
\hline Thhalv10000114m & AT2G22330.1 & CYP79B3 & cytochrome P450 79B3 & up & up & 5,92 & 0,000 & 5,05 & 0,000 & 16 & 7685 & 4205 \\
\hline Thhalv10008152m & AT1G18570.1 & AtMYB51,HIG1 & myb domain protein 51 & up & up & 5,68 & 0,000 & 4,31 & 0,000 & 164 & 13093 & 7845 \\
\hline Thhalv10024861m & AT4G39950.1 & CYP79B2 & cytochrome P450 79B2 & up & up & 5,06 & 0,000 & 5,38 & 0,000 & 254 & 24763 & 30609 \\
\hline Thhalv10007964m & AT1G21120.1 & IGMT2 & O-methyltransferase family protein & up & up & 4,93 & 0,000 & 2,70 & 0,001 & 434 & 9009 & 9298 \\
\hline Thhalv10018795m & AT1G74100.1 & ATST5A,SOT16 & sulfotransferase 16 & up & up & 4,54 & 0,000 & 4,45 & 0,000 & 665 & 26889 & 29842 \\
\hline Thhalv10024979m & AT4G37410.1 & CYP81F4 & cytochrome P450 81 F4 & up & up & 4,52 & 0,000 & 5,27 & 0,000 & 300 & 21717 & 48198 \\
\hline Thhalv10008073m & AT1G18590.1 & ATST5C,SOT17 & sulfotransferase 17 & up & up & 4,07 & 0,000 & 2,59 & 0,002 & 877 & 8651 & 4339 \\
\hline
\end{tabular}


Table 2 Analysis of transcript abundance changes of genes associated with the biosynthesis of defence-related metabolites (Continued)

\begin{tabular}{|c|c|c|c|c|c|c|c|c|c|c|c|c|}
\hline Thhalv10026067m & AT4G30530.1 & GGP1 & gammaglutamyl peptidase 1 & up & up & 3,31 & 0,000 & 3,55 & 0,000 & 5315 & 66528 & 98213 \\
\hline Thhalv10001994m & AT2G20610.1 & SUR1,ALF1,RTY1 & superroot1 & up & up & 3,22 & 0,000 & 2,48 & 0,000 & 14 & 28 & 23 \\
\hline Thhalv10018739m & AT1G76790.1 & IGMT5 & $\begin{array}{l}\text { O-methyltransferase family } \\
\text { protein }\end{array}$ & up & up & 2,63 & 0,001 & 3,03 & 0,000 & 1747 & 42057 & 94252 \\
\hline Thhalv10007574m & AT1G24100.1 & UGT74B1 & UDP-glucosyl transferase 74B1 & up & up & 2,44 & 0,000 & 2,15 & 0,000 & 1062 & 6408 & 5926 \\
\hline Thhalv10024981m & AT4G37430.1 & CYP81F1 & cytochrome P450 81 F1 & & up & 1,99 & 0,002 & 4,94 & 0,000 & 131 & 84 & 393 \\
\hline Thhalv10004064m & AT4G31500.1 & CYP83B1,SUR2 & cytochrome P450 83B1 & up & up & 1,70 & 0,001 & 2,06 & 0,000 & 2841 & 51916 & 96397 \\
\hline Thhalv10027443m & AT4G37400.1 & CYP81F3 & cytochrome P450 81 F3 & & up & $-3,97$ & 0,000 & 1,14 & 0,043 & 5 & 9 & 544 \\
\hline \multicolumn{13}{|c|}{ Phenylpropanoid biosynthesis } \\
\hline Thhalv10025563m & AT4G34230.1 & CAD5 & cinnamyl alcohol dehydrogenase 5 & up & up & 4,90 & 0,000 & 4,92 & 0,000 & 590 & 14915 & 11374 \\
\hline Thhalv10016314m & AT2G37040.1 & PAL1 & PHE ammonia lyase 1 & up & up & 4,54 & 0,000 & 4,32 & 0,000 & 5639 & 43919 & 53026 \\
\hline Thhalv10010153m & AT3G53260.1 & ATPAL2,PAL2 & PHE ammonia lyase 2 & up & up & 4,47 & 0,000 & 4,08 & 0,000 & 5516 & 24942 & 29448 \\
\hline Thhalv10016545m & AT2G30490.1 & C4H,CYP73A5 & cinnamate-4-hydroxylase & up & up & 4,47 & 0,000 & 3,92 & 0,000 & 3465 & 40914 & 24286 \\
\hline Thhalv10018849m & AT1G80820.1 & CCR2 & cinnamoyl coa reductase & up & up & 4,38 & 0,000 & 3,95 & 0,000 & 9 & 2796 & 6118 \\
\hline Thhalv10016544m & AT2G30490.1 & CYP73A5,REF3 & cinnamate-4-hydroxylase & up & up & 4,34 & 0,000 & 4,28 & 0,000 & 200 & 5351 & 6505 \\
\hline Thhalv10020406m & AT3G21230.1 & $4 C L 5$ & 4-coumarate:CoA ligase 5 & up & up & 2,86 & 0,000 & 1,30 & 0,000 & 160 & 961 & 882 \\
\hline Thhalv10001440m & AT2G43820.1 & SGT1,UGT74F2 & UDP-glucosyltransferase 74 F2 & up & up & 2,58 & 0,001 & 4,45 & 0,000 & 98 & 1100 & 1193 \\
\hline Thhalv10004662m & AT5G08640.1 & FLS1 & flavonol synthase 1 & up & up & 2,41 & 0,003 & 0,80 & 0,026 & 52 & 416 & 498 \\
\hline Thhalv10016538m & AT2G40890.1 & CYP98A3 & cytochrome P450, 98A3 & & & 2,32 & 0,001 & 2,10 & 0,000 & 1482 & 847 & 699 \\
\hline Thhalv10011357m & AT1G51680.3 & $4 \mathrm{CL} 1$ & 4-coumarate:CoA ligase 1 & up & up & 1,85 & 0,002 & 1,35 & 0,000 & 875 & 3071 & 2269 \\
\hline Thhalv10010658m & AT3G55120.1 & $\mathrm{A} 11, \mathrm{CFI}, \mathrm{TT} 5$ & Chalcone-flavanone isomerase & up & up & 1,76 & 0,001 & 2,04 & 0,000 & 112 & 352 & 573 \\
\hline Thhalv10024928m & AT4G36220.1 & CYP84A1,FAH1 & ferulic acid 5-hydroxylase 1 & up & up & 1,51 & 0,002 & 1,83 & 0,000 & 5064 & 5573 & 8972 \\
\hline Thhalv10026028m & AT4G34050.1 & CCOAOMT1 & SAM-dependent methyltransferase & up & & 0,99 & 0,010 & 0,04 & 0,779 & 851 & 3093 & 3791 \\
\hline Thhalv10000324m & AT3G21230.1 & $4 \mathrm{CL} 5$ & 4-coumarate:CoA ligase 5 & & up & 0,85 & 0,021 & 0,75 & 0,001 & 28 & 16 & 240 \\
\hline Thhalv10008111m & AT1G15950.1 & CCR1 & cinnamoyl coa reductase 1 & up & & 0,76 & 0,008 & 0,15 & 0,447 & 423 & 586 & 375 \\
\hline Thhalv10000513m & AT3G21230.1 & $4 \mathrm{CL} 5$ & 4-coumarate:CoA ligase 5 & & & 0,27 & 0,533 & $-0,03$ & 0,957 & 21 & 17 & 339 \\
\hline Thhalv10018769m & AT1G72680.1 & CAD1 & cinnamyl-alcohol dehydrogenase & & down & $-0,15$ & 0,461 & $-0,23$ & 0,050 & 859 & 635 & 666 \\
\hline Thhalv10022462m & AT1G65060.1 & $4 \mathrm{CL3}$ & 4-coumarate:CoA ligase 3 & & down & $-0,25$ & 0,141 & $-0,55$ & 0,004 & 113 & 23 & 15 \\
\hline Thhalv10020439m & AT3G21230.1 & $4 C L 5$ & 4-coumarate:CoA ligase 5 & down & down & $-0,83$ & 0,015 & $-0,93$ & 0,004 & 910 & 214 & 484 \\
\hline Thhalv10027317m & AT4G36220.1 & CYP84A1,FAH1 & ferulic acid 5-hydroxylase 1 & & & $-0,98$ & 0,338 & $-1,38$ & 0,170 & 1 & 1 & 3 \\
\hline Thhalv10013289m & AT5G07990.1 & CYP75B1,TT7 & cytochrome P450, 75B1 & & & $-1,26$ & 0,080 & $-1,16$ & 0,084 & 4 & 2 & 33 \\
\hline Thhalv10004668m & AT5G08640.1 & ATFLS1,FLS,FLS1 & flavonol synthase 1 & down & down & $-1,43$ & 0,005 & $-2,38$ & 0,002 & 137 & 17 & 41 \\
\hline Thhalv10005442m & AT1G43620.1 & TT15,UGT80B1 & UDP-Glycosyltransferase 80B1 & down & up & $-1,72$ & 0,000 & 0,34 & 0,008 & 1061 & 827 & 4174 \\
\hline Thhalv10014054m & AT5G08640.1 & FLS1 & flavonol synthase 1 & down & & $-2,67$ & 0,000 & $-2,92$ & 0,001 & 17 & 11 & 19 \\
\hline Thhalv10013745m & AT5G13930.1 & $\mathrm{CHS}, \mathrm{TT} 4$ & Chalcone and stilbene synth. Fam. & down & down & $-4,04$ & 0,000 & $-3,32$ & 0,005 & 214 & 11 & 200 \\
\hline
\end{tabular}


for the responses of orthologs of E. salsugineum genes we analysed by RT-qPCR (Figure 1). Moderate upregulation with respect to $0 \mathrm{~h}$, peaking at $3 \mathrm{~h}$ for AtASA1 (5.0fold) and AtPEN2 (2.0-fold), and at $6 \mathrm{~h}$ for AtIGMT5 (3.6-fold) and AtBGLU18 (3.3-fold) was observed. More generally, we surveyed these data for core indole glucosinolate biosynthetic genes and again observed only modest transcript induction $6 \mathrm{~h}$ after UV treatment (less than 5-fold upregulation of CYP83B1, SUR1, GGP1, SOT16 and UGT74B1). In contrast, the camalexin biosynthetic genes CYP71B15 and CYP71A13 were induced approximately 121 -fold and 66-fold, respectively [41]. These differential responses are consistent with the proposed phytoalexin biosynthetic pathways in the two species.

In Arabidopsis, unmodified indole glucosinolate is methoxylated in response to pathogen infection, involving members of the CYP81F family and indole glucosinolate methyl transferases (IGMTs) [42]. E. salsugineum contains five CYP81F members, due to an additional gene copy in the CYP81F1/3/4 cluster. For three of these genes, microarray and RNAseq data were obtained and matched. Based on its expression pattern, EsCYP81F4 (Thhalv10024979m) is a candidate gene for catalysing N-hydroxylation of 3indolylmethylglucosinolate in the biosynthesis of Eutrema phytoalexins. EsCYP81F3 (Thhalv10027443m) was induced by $\mathrm{AgNO}_{3}$ but not by UV light. Also, EsIGMT5, highly expressed in response to stress treatment (Table 2 , Figure 1), is a candidate for involvement in the biosynthesis of N-methoxylated indolic compounds.

In response to pathogen infection, in Arabidopsis indole glucosinolates are degraded to bioactive compounds by the $\beta$-glucosidase PEN2 (BGLU26) [43,44]. We hypothesize that $\beta$-glucosidases are also involved in the biosynthesis of Eutrema phytoalexins. A number of $\beta$-glucosidase-encoding genes were significantly upregulated in response to $\mathrm{AgNO}_{3}$ and UV challenge (Table 3), including EsPEN2 (Thhalv10001354m), EsBGLU18-1 (Thhalv10011384m), and EsBGLU18-2 (Thhalv100113 $85 \mathrm{~m})$. The time course of induction of these genes was monitored by quantitative RT-PCR and strong induction responses to $\mathrm{AgNO}_{3}$ and UV treatment were confirmed (Figure 1). In conclusion, the Eutrema orthologs of PEN2 (BGLU26) and BGLU18 are candidates for an involvement in phytoalexin biosynthesis.

In response to UV light and $\mathrm{AgNO}_{3}$, most genes involved in aliphatic glucosinolate biosynthesis were strongly downregulated, with the exception of the putative orthologs of flavin-containing monooxygenase (FMO) genes encoding glucosinolate S-oxygenases (Table 2), probably resulting in a metabolic shift towards indolic and oxidized aliphatic glucosinolates. Based on homology and chromosomal position Thhalv10008073m is orthologous to AtSOT17/AtST5c (At1g18590), encoding a sulfotransferase with a preference for aliphatic desulfoglucosinolates as substrates $[45,46]$. Here, we observed strong transcriptional upregulation of EsSOT17 (Thhalv10008073m) in response to $\mathrm{UV}$ irradiation and $\mathrm{AgNO}_{3}$ treatment, similar to genes involved in indole glucosinolate biosynthesis. We speculate that in the two species the two orthologs have acquired different substrate specificities and that the Eutrema gene functions in indole glucosinolate biosynthesis. The two other confirmed desulfoglucosinolate sulfotransferases AtSOT18/AtST5b and AtSOT16/AtST5a have probably retained their function in aliphatic and indole glucosinolate biosynthesis, respectively.

\section{WRKY transcription factors}

In Arabidopsis, WRKY transcription factors play an essential role in the regulation of phytoalexin responses. Our data show that also in Eutrema several WRKY genes are upregulated, including the orthologs of WRKY40, WRKY75, WRKY33, WRKY6, WRKY51 and WRKY18 (Table 4). WRKY18 and WRKY40 are central regulators of indole glucosinolate modification in response to pathogens [47]. WRKY6 is associated with both senescenceand defence-related processes [48] and WRKY75, besides its role in phosphate acquisition [49], is also linked to senescence and pathogen defence [50,51]. WRKY51 plays a role in modulation of salicylate- and jasmonate signalling in defence [52]. In summary, these transcriptional changes indicate that also in Eutrema WRKYs are crucial for induced metabolic defence.

\section{EsWRKY33 complements camalexin deficiency in an Arabidopsis WRKY mutant}

In Arabidopsis, WRKY33 is an essential regulator of camalexin biosynthesis and directly binds to the promoter of CYP71B15 (PAD3) [19]. Accordingly, its expression is induced by Pathogen-associated molecular patterns (PAMPs) and it is important for resistance against necrotrophic fungal pathogens [53-56]. Camalexin has not been detected in Eutrema and it does not contain a clear ortholog of CYP71B15. The closest CYP71B15 homolog in E. salsugineum shares only $66.7 \%$ identical amino acids. Nevertheless, Es WRKY33 is strongly upregulated upon phytoalexin inducing conditions (Figure 1; Table 4).

We investigated whether EsWRKY33 can functionally replace AtWRKY33 as a positive regulator of camalexin biosynthesis and expressed EsWRKY33 in the Arabidopsis wrky33-1 mutant [54]. While in wrky33 leaves camalexin levels were significantly reduced in relation to wild type, wild type levels were restored in the complementing line (Figure 4). This indicates that even though Eutrema does not synthesize camalexin, EsWRKY33 can 
Table 3 Analysis of transcript abundance changes of genes encoding $\beta$-glucosidases

\begin{tabular}{|c|c|c|c|c|c|c|c|c|c|c|c|}
\hline \multirow[t]{2}{*}{ Transcript ID } & \multirow[t]{2}{*}{ Best Ath hit } & \multirow{2}{*}{$\begin{array}{l}\text { Gene } \\
\text { symbol }\end{array}$} & \multirow[t]{2}{*}{ UV } & \multirow[t]{2}{*}{$\mathrm{Ag}^{+}$} & \multirow{2}{*}{$\begin{array}{l}\text { Fold } \\
\text { change } \\
\log 2 \\
\text { (UV/n.i.) }\end{array}$} & \multirow{2}{*}{$\begin{array}{l}\text { FDR-p-value } \\
\text { test }\end{array}$} & \multirow{2}{*}{$\begin{array}{l}\text { Fold } \\
\text { change } \\
\text { log2 } \\
\text { (Ag/n.i.) }\end{array}$} & \multirow{2}{*}{$\begin{array}{l}\text { FDR-p-value } \\
\text { test }\end{array}$} & \multicolumn{3}{|c|}{ RNAseq Unique reads } \\
\hline & & & & & & & & & n.i. & UV & AgNO3 \\
\hline Thhalv10006515m & AT4G27830.1 & BGLU10 & up & & 1,80 & 0,001 & $-1,56$ & 0,000 & 3 & 30 & 6 \\
\hline Thhalv10006510m & AT4G27830.1 & BGLU10 & up & & 1,42 & 0,000 & $-1,94$ & 0,000 & 40 & 389 & 105 \\
\hline Thhalv10005908m & AT4G27830.1 & BGLU10 & down & & $-2,08$ & 0,000 & $-1,85$ & 0,000 & 217 & 127 & 587 \\
\hline Thhalv10001447m & AT2G44450.1 & BGLU15 & up & up & 1,14 & 0,003 & 2,08 & 0,000 & 2 & 20 & 29 \\
\hline Thhalv10011384m & AT1G52400.1 & BGLU18 & down & up & $-5,32$ & 0,000 & 0,47 & 0,000 & 4823 & 2272 & 195299 \\
\hline Thhalv10011385m & AT1G52400.1 & BGLU18 & & & $-7,87$ & 0,000 & 0,41 & 0,279 & 2 & 6 & 1246 \\
\hline Thhalv10020508m & AT3G09260.1 & BGLU23,PYK10 & & up & 0,18 & 0,650 & 1,20 & 0,045 & 19 & 106 & 861 \\
\hline Thhalv10020496m & AT3G03640.1 & BGLU25,GLUC & & up & $-0,13$ & 0,582 & 1,46 & 0,001 & 415 & 811 & 1634 \\
\hline Thhalv10001354m & AT2G44490.1 & BGLU26,PEN2 & up & up & 1,94 & 0,000 & 1,70 & 0,000 & 2401 & 11117 & 21129 \\
\hline Thhalv10002501m & AT4G22100.1 & BGLU3 & & & $-1,19$ & 0,036 & $-0,22$ & 0,547 & 2 & 20 & 542 \\
\hline Thhalv10004297m & AT4G22100.1 & BGLU3 & down & down & $-1,30$ & 0,000 & $-2,00$ & 0,000 & 11 & 3 & 8 \\
\hline Thhalv10028552m & AT4G22100.1 & BGLU3 & & & $-1,85$ & 0,000 & $-1,19$ & 0,000 & 85 & 89 & 170 \\
\hline Thhalv10002493m & AT4G22100.1 & BGLU3 & down & & $-2,57$ & 0,000 & $-1,31$ & 0,001 & 100 & 92 & 571 \\
\hline Thhalv10005858m & AT3G60140.1 & BGLU30,SRG2 & & up & 0,50 & 0,566 & 6,19 & 0,000 & 5 & 18 & 344 \\
\hline Thhalv10018387m & AT5G24550.1 & BGLU32 & up & up & 5,96 & 0,000 & 6,62 & 0,000 & 729 & 31451 & 52650 \\
\hline Thhalv10002474m & AT5G26000.1 & BGLU38,TGG1 & & & $-0,37$ & 0,529 & $-0,38$ & 0,598 & 7 & 0 & 2 \\
\hline Thhalv10004165m & AT5G26000.1 & BGLU38,TGG1 & & & $-0,68$ & 0,303 & $-0,73$ & 0,375 & 11 & 1 & 2 \\
\hline Thhalv10003954m & AT5G26000.1 & BGLU38,TGG1 & & & $-1,29$ & 0,085 & $-0,88$ & 0,219 & 6 & 0 & 10 \\
\hline Thhalv10007404m & AT1G26560.1 & BGLU40 & up & up & 2,11 & 0,000 & 2,54 & 0,000 & 167 & 652 & 1015 \\
\hline Thhalv10027734m & AT5G36890.1 & BGLU42 & down & & $-1,09$ & 0,000 & 0,24 & 0,001 & 773 & 351 & 731 \\
\hline Thhalv10020536m & AT3G18080.1 & BGLU44 & & & 1,81 & 0,002 & 1,01 & 0,006 & 8 & 5 & 3 \\
\hline Thhalv10023411m & AT1G61820.1 & BGLU46 & up & up & 2,81 & 0,005 & 7,02 & 0,000 & 1 & 157 & 964 \\
\hline
\end{tabular}


Table 4 Analysis of transcript abundance changes of genes encoding WRKY transcription factors

\begin{tabular}{|c|c|c|c|c|c|c|c|c|c|c|c|}
\hline \multirow[t]{2}{*}{ Transcript ID } & \multirow[t]{2}{*}{ Best Ath hit } & \multirow[t]{2}{*}{ WRKY } & \multirow[t]{2}{*}{ UV } & \multirow[t]{2}{*}{$\mathrm{Ag}^{+}$} & \multirow{2}{*}{$\begin{array}{l}\text { Fold } \\
\text { change } \\
\text { log2 } \\
\text { (UV/n.i.) }\end{array}$} & \multirow{2}{*}{$\begin{array}{l}\text { FDR-p-value } \\
\text { test }\end{array}$} & \multirow{2}{*}{$\begin{array}{l}\text { Fold } \\
\text { change } \\
\log 2 \\
(\mathrm{Ag} / \text { n.i.) }\end{array}$} & \multirow{2}{*}{$\begin{array}{l}\text { FDR-p-value } \\
\text { test }\end{array}$} & \multicolumn{3}{|c|}{ RNAseq Unique reads } \\
\hline & & & & & & & & & n.i. & UV & AgNO3 \\
\hline Thhalv10002516m & AT2G04880.2 & 1 & down & down & $-0,16$ & 0,026 & $-0,39$ & 0,000 & 170 & 79 & 89 \\
\hline Thhalv10012829m & AT5G56270.1 & 2 & & up & 0,35 & 0,144 & 0,37 & 0,014 & 742 & 649 & 786 \\
\hline Thhalv10004015m & AT2G03340.1 & 3 & & & $-0,27$ & 0,315 & $-0,02$ & 0,923 & 746 & 866 & 648 \\
\hline Thhalv10007428m & AT1G13960.1 & 4 & up & up & 2,27 & 0,000 & 2,56 & 0,000 & 1482 & 1556 & 2016 \\
\hline Thhalv10023390m & AT1G62300.1 & 6 & up & up & 4,84 & 0,000 & 5,03 & 0,000 & 83 & 7722 & 9852 \\
\hline Thhalv10025630m & AT4G24240.1 & 7 & & & $-0,25$ & 0,122 & $-0,21$ & 0,241 & 384 & 161 & 260 \\
\hline Thhalv10025646m & AT4G31550.1 & 11 & up & up & 1,93 & 0,001 & 3,53 & 0,000 & 120 & 700 & 3144 \\
\hline Thhalv10000270m & AT2G23320.1 & 15 & up & up & 4,43 & 0,000 & 3,38 & 0,000 & 757 & 4909 & 2951 \\
\hline Thhalv10001165m & AT5G45050.2 & 16 & & & $-0,22$ & 0,641 & 0,64 & 0,116 & 5963 & 1782 & 5082 \\
\hline Thhalv10000242m & AT2G24570.1 & 17 & up & up & 1,64 & 0,001 & 2,40 & 0,000 & 300 & 1089 & 2481 \\
\hline Thhalv10025785m & AT4G31800.1 & 18 & up & up & 2,15 & 0,002 & 3,61 & 0,000 & 173 & 1582 & 2793 \\
\hline Thhalv10024810m & AT4G26640.2 & 20 & & down & $-0,17$ & 0,133 & $-0,38$ & 0,029 & 646 & 333 & 300 \\
\hline Thhalv10013115m & AT4G26640.2 & 20 & down & & $-1,46$ & 0,035 & 0,40 & 0,637 & 367 & 124 & 210 \\
\hline Thhalv10016852m & AT2G30590.1 & 21 & up & & 0,69 & 0,003 & 0,40 & 0,001 & 8 & 12 & 7 \\
\hline Thhalv10028843m & AT4G01250.1 & 22 & up & up & 2,88 & 0,000 & 4,37 & 0,000 & 7 & 50 & 211 \\
\hline Thhalv10016764m & AT2G30250.1 & 25 & up & up & 1,79 & 0,001 & 2,06 & 0,000 & 239 & 1182 & 1844 \\
\hline Thhalv10017017m & AT2G30250.1 & 25 & up & up & 1,55 & 0,010 & 2,22 & 0,000 & 22 & 120 & 136 \\
\hline Thhalv10013872m & AT5G52830.1 & 27 & & down & 0,72 & 0,036 & $-1,29$ & 0,005 & 48 & 27 & 10 \\
\hline Thhalv10025799m & AT4G23550.1 & 29 & & & 1,71 & 0,009 & $-0,42$ & 0,428 & 18 & 16 & 23 \\
\hline Thhalv10025126m & AT4G30935.1 & 32 & & down & $-0,17$ & 0,244 & $-0,33$ & 0,049 & 1203 & 391 & 285 \\
\hline Thhalv10016542m & AT2G38470.1 & 33 & up & up & 5,31 & 0,000 & 4,37 & 0,000 & 325 & 15433 & 11838 \\
\hline Thhalv10021115m & AT3G04670.1 & 39 & & up & 1,14 & 0,001 & 1,98 & 0,000 & 169 & 143 & 195 \\
\hline Thhalv10018925m & AT1G80840.1 & 40 & up & up & 6,44 & 0,000 & 4,50 & 0,000 & 23 & 7398 & 7919 \\
\hline Thhalv10028794m & AT4G1 1070.1 & 41 & up & up & 4,24 & 0,000 & 3,22 & 0,000 & 6 & 46 & 85 \\
\hline Thhalv10001568m & AT2G46400.1 & 46 & up & up & 3,15 & 0,000 & 4,01 & 0,000 & 72 & 925 & 2639 \\
\hline Thhalv10005029m & AT5G26170.1 & 50 & & up & 0,17 & 0,680 & 1,30 & 0,005 & 41 & 67 & 93 \\
\hline Thhalv10004930m & AT5G64810.1 & 51 & up & up & 4,29 & 0,000 & 4,30 & 0,000 & 55 & 1520 & 1853 \\
\hline Thhalv10016713m & AT2G40750.1 & 54 & up & up & 1,27 & 0,005 & 2,38 & 0,000 & 407 & 1840 & 3229 \\
\hline Thhalv10017926m & AT2G40740.1 & 55 & up & up & 6,27 & 0,000 & 4,45 & 0,000 & 31 & 241 & 411 \\
\hline Thhalv10018976m & AT1G69310.1 & 57 & & & 0,12 & 0,559 & 0,28 & 0,173 & 410 & 196 & 249 \\
\hline Thhalv10000288m & AT2G25000.1 & 60 & down & down & $-2,14$ & 0,000 & $-0,70$ & 0,002 & 99 & 37 & 90 \\
\hline Thhalv10006157m & AT3G58710.1 & 69 & up & & $-0,52$ & 0,162 & 1,54 & 0,001 & 34 & 48 & 194 \\
\hline Thhalv10006146m & AT3G56400.1 & 70 & up & up & 1,66 & 0,002 & 4,40 & 0,000 & 216 & 2525 & 12004 \\
\hline Thhalv10013146m & AT5G15130.1 & 72 & & up & 0,30 & 0,450 & 3,54 & 0,000 & 1 & 1 & 25 \\
\hline Thhalv10014943m & AT5G13080.1 & 75 & up & up & 6,26 & 0,000 & 7,02 & 0,000 & 9 & 1076 & 1311 \\
\hline
\end{tabular}


act as a positive regulator of camalexin biosynthesis in Arabidopsis.

\section{Conclusions}

In E. salsugineum, UV irradiation or heavy metal application resulted in substantial transcriptional reprogramming consistent with the induction of defence responses. Photosynthesis and starch synthesis were transcriptionally downregulated, while processes providing precursors for aromatic defence metabolites and cell wall compounds were transcriptionally induced. Strikingly, a shift in expression is observed from orthologs of genes for the biosynthesis of aliphatic glucosinolates, probably functioning primarily in insect defence, to orthologs of genes for the biosynthesis of indole glucosinolates, serving as precursors of phytoalexins.

WRKY33 is an essential regulator of the camalexin biosynthetic gene CYP71B15 (PAD3) [19], for which there is probably no functional homolog in E. salsugineum, consistent with the absence of camalexin in this species [12]. Nevertheless, there is a putative Eutrema WRKY33 ortho$\log$, which is strongly upregulated under phytoalexin inducing conditions. EsWRKY33 was functionally tested and shown to complement camalexin deficiency in an Atwrky33 mutant. We hypothesize that regulatory mechanisms for phytoalexin induction are conserved among members of the Brassicaceae, while the individual chemical structures have strongly diversified.

\section{Methods}

\section{Plant growth conditions and stress treatments}

After 10 days (E. salsugineum) or two days (A. thaliana) of stratification at $6^{\circ} \mathrm{C}$, plants were grown in a growth chamber at a $12 / 12 \mathrm{~h}$ photoperiod at a light intensity of 80 to $100 \mu \mathrm{mol} \mathrm{m}^{-2} \mathrm{~s}^{-1}$ at $21^{\circ} \mathrm{C}$ and $40 \%$ relative humidity. For stress treatment leaves were sprayed with $5 \mathrm{mM}$ $\mathrm{AgNO}_{3}$ or placed under a UV lamp (Desaga UVVIS, $\lambda=$ $254 \mathrm{~nm}, 8 \mathrm{~W}$ ) at a distance of $20 \mathrm{~cm}$ and radiated for 2 h. For Botrytis cinerea infection a spore suspension (strain B05.10, $2 \times 10^{5}$ spores per $\mathrm{ml}$ ) was sprayed on the leaf surface.

\section{RNA isolation, CDNA preparation and RT-qPCR}

RNA extraction, cDNA synthesis and RT-qPCR, performed with the SYBRGreen/Light Cycler system (Roche), has been described previously [57]. The following primers were used:

AtActin1: 5'TGGAACTGGAATGGTTAAGGCTGG3' and 5'TCTCCAGAGTCGAGCACAATACCG3' AtGAPC: 5'GCACCTTTCCGACAGCCTTG3' and 5' ATTAGGATCGGAATCAACGG3

EsActin1: 5'TGGAACTGGAATGGTTAAGGCTGG3' and 5'TCTCCAGAGTCGAGCACAATACCG3'
EsYLS8: 5'GCGATTCTGGCTGAGGAAGA3' and 5' CTTCCTTGCACCACGGTAGA3'

EsPP2AA2: 5'TGCTGAAGATAGGCACTGGA3' and 5'CATTGAATTTGATGTTGGGAAC3'

EsASA1: 5'ATGTCTAGCGTTGGTCGTTATAGCG3' and 5'CTTGACCACAGCCTCCTTGTACTCT3'

EsIGMT5: 5'AGTGCCAAGTCGTTGATGGT3' and 5' TTGATACCCTTGATGTTTGGA3'

EsBGLU18-1: 5'AGAGGACCTTGGAGACCTTC3'

and 5'AGTTCTTCCCTCACTAACTTGGA3'

EsBGLU18-2: 5'CCTACTCGTGCTCTACTGGA3' and 5'TCCCGGCTTAAGGAAATCAGA3'

EsPEN2: 5'CCAACAGGACTCAGAAACGT3' and 5' GCAGTGACAACGAACAAGCT3'

Es WRKY33: 5'TATCCATTCACAGGAACAACAG

AG3' and 5'GGATGGTTATGGCTTCCCTT3'.

Expression values of candidate genes were normalized to the geometric mean of three reference genes [58] (EsActin1, EsYELLOW-LEAF-SPECIFIC GENE 8 (ESYLS8), and ESPROTEIN PHOSPHATASE $2 A$ SUBUNIT A2 (EsPP2AA2)). Expression level of EsWRKY33 in the $A$. thaliana background was normalized to AtActin1 and AtGAPC.

\section{RNAseq setup and analysis}

Total RNA was isolated from three biological replicates of either control leaves or from leaves treated either with UV light for $2 \mathrm{~h}$ followed by $6 \mathrm{~h}$ recovery, leaves sprayed with $5 \mathrm{mM} \mathrm{AgNO}_{3}$ and incubated $8 \mathrm{~h}$, or $48 \mathrm{~h}$ after infection of plants with $B$. cinerea, using the NucleoSpin ${ }^{\circ}$ RNA II Kit (Machery-Nagel). Single-end cDNA libraries were prepared and sequenced using Illumina HiSeq 2000 technology at LGC Genomics [59] to obtain 50 bp reads. Demultiplexing was done using Illumina's CASAVA software [60]. Reads were adapter-clipped and reads shorter than 20 bases were discarded. Read quality was assessed using FASTQC [61]. Table 1 lists the resulting number of reads used for analyses.

CLC Genomics workbench Version 6.5.1 [62] was used for RNAseq analysis including mapping to the Eutrema reference transcriptome [9] using default settings, allowing for at most two mismatches and a maximum of 10 transcript hits per read and generation of RPKM value statistic. Differential gene expression was detected using Fisher exact tests based on mapped read counts and with FDR-based correction for multiple testing errors [63]. Fold changes were computed using RPKM-values.

\section{Microarray setup and statistical analysis}

For each treatment, four biological replicates were investigated, generated from pooled tissues of 4 plants. Total RNA was extracted with NucleoSpin ${ }^{\circ}$ RNA II Kit (Machery-Nagel). After DNase treatment, concentration and 


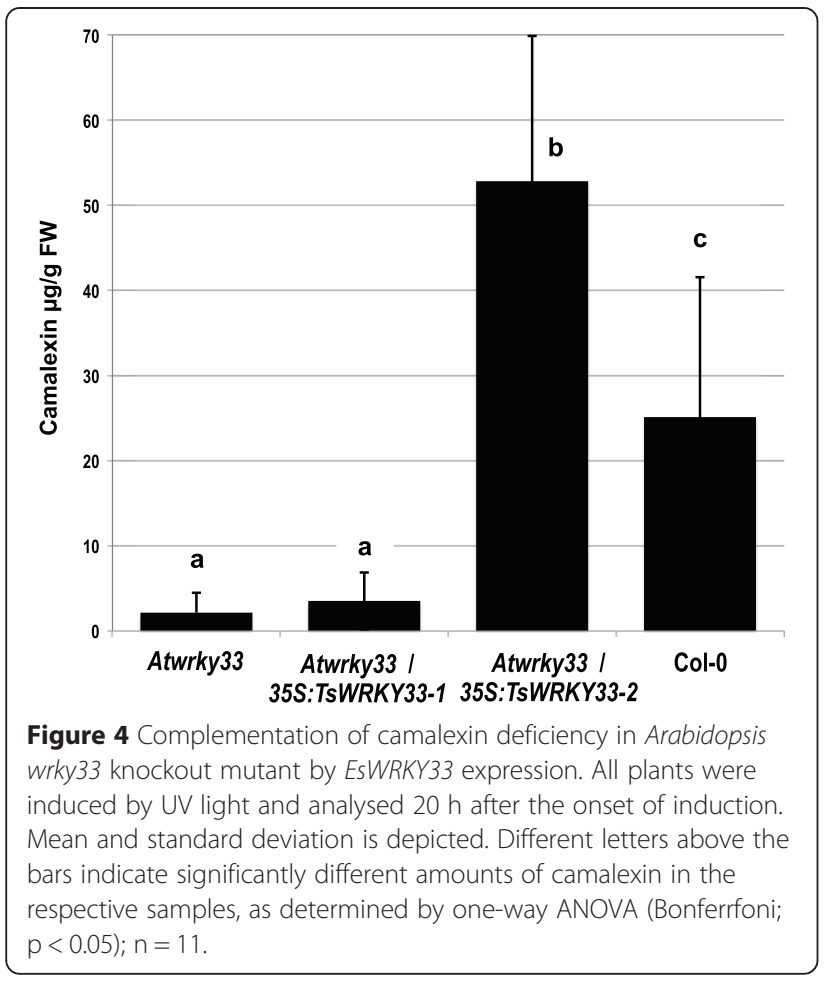

quality of extracted RNA was measured photometrically and with a Bioanalyzer (Agilent Technologies, Santa Clara, CA). Samples were hybridized to Agilent $8 \times 60 \mathrm{k}$ microarrays by OakLabs GmbH [64]. The arrays contain 42,562 oligonucleotide probes and are based on the recently developed Agilent $4 \times 44 \mathrm{k}$ Eutrema array [9]

Raw hybridization signals were quantile-normalized and log-base-2 transformed. Differential gene expression was assessed using ANOVA across all conditions and repeats and t-test statistic for pairwise comparisons with FDR-multiple testing correction [63]. Differential gene expression was mapped to metabolic pathways using the MAPMAN software [20].

\section{RNAseq and microarray data match, functional} annotation/candidate orthologs in Arabidopsis thaliana Mapping of array probe identifiers to reference transcriptome identifiers was based on sequence matches using BLASTN with an E-value cutoff of 1E-05. Candidate ortholog genes in Arabidopsis thaliana were identified as best sequence identity hits using BLASTN with the same cutoff. The set of representative Arabidopsis transcripts available from TAIR10 [65] was used.

\section{Generation of WRKY33 complementation lines}

EsWRKY33 (Thhalv10016542m) coding sequence was amplified from cDNA (E. salsugineum leaves, $5 \mathrm{~h}$ after UV treatment) using the primer pair 5'GGCTTAAUA 'TGGCTGCTTCTTCTCTTC3' and 3'GGTTTAAUT
CACGACAAAAACGAATCAAA5' and cloned into pCAMBIA330035Su via USER technology [66]. After confirmation of the correct cDNA sequence, Agrobacterium-mediated transformation of Arabidopsis wrky33-1 knockout mutant (SALK_006603; [54]) was performed via floral-dipping, and successful transformants were confirmed by BASTA resistance of the seedlings and by PCR analysis. Primary transformants were analysed for EsWRKY33 expression by RT-qPCR. One low (\#1, 0.48 $\pm 0.26 \mathrm{fg} / \mathrm{fg}$ AtActin1, $0.11 \pm 0.09 \mathrm{fg} / \mathrm{fg}$ GAPC) and one high (\#2, $16.2 \pm 7.8 \mathrm{fg} / \mathrm{fg}$ AtActin1, $2.8 \pm 1.7 \mathrm{fg} / \mathrm{fg}$ $G A P C)$ expression line was selected for phenotype analysis.

\section{Metabolite analysis}

Camalexin formation was induced in six-week old $A$. thaliana plants by treatment with UV (see above). Camalexin was isolated $20 \mathrm{~h}$ after the onset of induction and quantified applying HPLC with fluorescence detection as described [67]. For monitoring wasalexin A formation, E. salsugineum leaves were treated with either UV light for $2 \mathrm{~h}$ followed by $22 \mathrm{~h}$ incubation, sprayed with $5 \mathrm{mM} \mathrm{AgNO}_{3}$ and incubated $24 \mathrm{~h}$, or sprayed with B. cinerea spore suspension and incubated $48 \mathrm{~h}$. Plant material was frozen in liquid nitrogen. Leaves were ground and $900 \mu \mathrm{l}$ methanol were added. The samples were incubated at room temperature for $30 \mathrm{~min}$ under agitation, centrifuged for $15 \mathrm{~min}$ at $14,000 \mathrm{rpm}$ and the supernatant was transferred to a new tube. To increase the yield of metabolites the extraction was repeated once and supernatants were combined. The solvent was evaporated completely (SA-Speed Concentrator, H.Saur Laborbedarf) and metabolites were dissolved in $400 \mu \mathrm{l}$ $100 \%$ methanol. Quantification of Wasalexin A was done via reverse-phase HPLC (Göhler Multohigh100 RP-18, $5 \mu \mathrm{m}, 250 \mathrm{mmx} 4 \mathrm{~mm}$; flow rate: $1 \mathrm{ml} / \mathrm{min}$; solvents: acetonitrile and $0.3 \%$ formic acid in $\mathrm{H}_{2} \mathrm{O} ; 20 \%$ acetonitrile for $2 \mathrm{~min}$, followed by a $17 \mathrm{~min}$ linear gradient to $70 \%$ acetonitrile and then $3 \mathrm{~min}$ to $100 \%$ acetonitrile). The peaks at $20.2 \mathrm{~min}$ and $21.5 \mathrm{~min}\left(\mathrm{OD}_{\max }: 362 \mathrm{~nm}\right)$ were identified as Wasalexin B and Wasalexin A, respectively by comparison with authentic standard with respect to retention time and UV spectrum.

\section{Availability of supporting data}

All curated supporting data are included as additional files. The raw RNAseq data have been deposited in the National Center for Biotechnology Information (NCBI) Sequence Read Archive (SRA) database under the accession number SRP048695. Microarray data was deposited at Gene Expression Omnibus (GEO) database under the accession numbers GSM1530883 to GSM1530894 (platform accession GPL19319). 


\section{Additional files}

Additional file 1: Figure S1. Induction of wasalexin in response to UV light, $\mathrm{AgNO}_{3}$ and $B$. cinerea. Leaf extracts ( $24 \mathrm{~h}$ after onset of induction by UV or $\mathrm{AgNO}_{3}$ or $48 \mathrm{~h}$ after fungal infection) and authentic standard were analysed by HPLC (see Methods section). Representative chromatograms at $363 \mathrm{~nm}$ are shown.

Additional file 2: Figure S2. Venn diagram representing uniquely mapped transcripts identified in RNAseq of the four CDNA libraries.

Additional file 3: Table S1. Complete dataset for RNAseq analysis. Additional file 4: Table S2. Complete dataset for microarray analysis. Additional file 5: Figure S3. Correlation of log-fold changes between RNAseq and array data after matching. A: UV versus not induced (n.i.). B: $\mathrm{AgNO}_{3}$ versus n.i.

Additional file 6: Table S3. Complete dataset for 14706 genes, for which both array and RNAseq data is available.

Additional file 7: Figure S4. UV- and $\mathrm{AgNO}_{3}$-responsiveness of genes differentially regulated in other studies. A: 81 drought and 59 cold regulated E. salsugineum genes (Wong et al. [32]) B: Identified putative orthologs of A. thaliana genes upregulated in response to both $B$. cinerea and oxidative stress (115 identified, 7 unchanged in response to UV or $\mathrm{Ag}^{+}$).

\section{Abbreviations}

ANOVA: Analysis of variance; FDR: False discovery rate; JGl: Joint Genome Institute; RPKM: Reads per kilobase of transcript per million reads mapped; RT-qPCR: Reverse transcription quantitative polymerase chain reaction.

\section{Competing interests}

The authors declare that they have no competing interests.

\section{Authors' contributions}

SM conducted the majority of experiments and supported drafting the manuscript. DW carried out bioinformatic and statistical analysis. TM analysed WRKY33 function. DKH provided unpublished data and supported conceptualising the project. EG supervised the project, supported experiment design and analysis, and wrote the draft of the manuscript. All authors read, revised, and approved the manuscript.

\section{Acknowledgements}

We kindly thank Prof. Soledade Pedras for providing Wasalexin A standard, Prof. Barbara Halkier for providing pCAMBIA330035Su, and Prof. Paul Tudzynski for providing Botrytis cinerea B05.10. We thank Alexandra Chapman for assisting in establishment of Eutrema work and Heidi Miller-

Mommerskamp for plant propagation. This work has been supported by the Deutsche Forschungsgemeinschaft (DFG), grants 346/7 and 346/5 (Heisenberg fellowship to E.G.), and the Hans-Fischer-Gesellschaft für Bioorganische Chemie.

\section{Author details}

${ }^{1}$ Lehrstuhl für Genetik, Technische Universität München, D-85354 Freising, Germany. ${ }^{2}$ Max-Planck-Institut für Molekulare Pflanzenphysiologie, 14476 Potsdam, Germany.

Received: 27 February 2015 Accepted: 24 April 2015

Published online: 12 June 2015

\section{References}

1. Pedras MS, Yaya EE, Glawischnig E. The phytoalexins from cultivated and wild crucifers: chemistry and biology. Nat Prod Rep. 2011;28:1381-405.

2. Böttcher C, Westphal L, Schmotz C, Prade E, Scheel D, Glawischnig E. The multifunctional enzyme, CYP71B15 (PHYTOALEXIN DEFICIENT 3), converts cysteine-indole-3-acetonitrile to camalexin in the indole-3-acetonitrile metabolic network of Arabidopsis thaliana. Plant Cell. 2009;21:1830-45.

3. Böttcher C, Chapman A, Fellermeier F, Choudhary M, Scheel D, Glawischnig E. The biosynthetic pathway of indole-3-carbaldehyde and indole-3carboxylic acid derivatives in Arabidopsis thaliana. Plant Physiol. 2014;165(2):841-53.

4. Rauhut T, Glawischnig E. Evolution of camalexin and structurally related indolic compounds. Phytochemistry. 2009;68:401-6.
5. Kliebenstein DJ, Rowe HC, Denby KJ. Secondary metabolites influence Arabidopsis/Botrytis interactions: variation in host production and pathogen sensitivity. Plant J. 2005:44:25-36.

6. Amtmann A. Learning from evolution: Thellungiella generates new knowledge on essential and critical components of abiotic stress tolerance in plants. Mol Plant. 2009;2(1):3-12.

7. Wu HJ, Zhang Z, Wang JY, Oh DH, Dassanayake M, Liu B, et al. Insights into salt tolerance from the genome of Thellungiella salsuginea. Proc Natl Acad Sci U S A. 2012;109(30):12219-24.

8. Yang $\mathrm{R}$, Jarvis $\mathrm{DE}$, Chen $\mathrm{H}$, Beilstein MA, Grimwood J, Jenkins J, et al. The reference genome of the halophytic plant Eutrema salsugineum. Front Plant Sci. 2013;4:46.

9. Lee YP, Giorgi FM, Lohse M, Kvederaviciute K, Klages S, Usadel B, et al. Transcriptome sequencing and microarray design for functional genomics in the extremophile Arabidopsis relative Thellungiella salsuginea (Eutrema salsugineum). BMC Genomics. 2013;14:793.

10. Champigny MJ, Sung WW, Catana V, Salwan R, Summers PS, Dudley SA, et al. RNA-Seq effectively monitors gene expression in Eutrema salsugineum plants growing in an extreme natural habitat and in controlled growth cabinet conditions. BMC Genomics. 2013;14:578.

11. Pang Q, Chen S, Li L, Yan X. Characterization of glucosinolate-myrosinase system in developing salt cress Thellungiella halophila. Physiol Plant. 2009;136(1):1-9.

12. Pedras MS, Adio AM. Phytoalexins and phytoanticipins from the wild crucifers Thellungiella halophila and Arabidopsis thaliana: rapalexin A, wasalexins and camalexin. Phytochemistry. 2008;69(4):889-93.

13. Pedras MS, Zheng QA. Metabolic responses of Thellungiella halophila/ salsuginea to biotic and abiotic stresses: metabolite profiles and quantitative analyses. Phytochemistry. 2010;71(5-6):581-9.

14. Koch MA, German DA. Taxonomy and systematics are key to biological information: Arabidopsis, Eutrema (Thellungiella), Noccaea and Schrenkiella (Brassicaceae) as examples. Front Plant Sci. 2013;4:267.

15. Brown PD, Tokuhisa JG, Reichelt M, Gershenzon J. Variation of glucosinolate accumulation among different organs and developmental stages of Arabidopsis thaliana. Phytochemistry. 2003;62(3):471-81.

16. Petersen $\mathrm{BL}$, Chen $\mathrm{S}$, Hansen $\mathrm{CH}$, Olsen $\mathrm{CE}$, Halkier BA. Composition and content of glucosinolates in developing Arabidopsis thaliana. Planta. 2012;214(4):562-71.

17. Glawischnig E, Hansen BG, Olsen CE, Halkier BA. Camalexin is synthesized from indole-3-acetaldoxime, a key branching point between primary and secondary metabolism in Arabidopsis. Proc Natl Acad Sci U S A. 2004;101:8245-50.

18. Pedras MS, Yaya EE, Hossain S. Unveiling the phytoalexin biosynthetic puzzle in salt cress: unprecedented incorporation of glucobrassicin into wasalexins A and B. Org Biomol Chem. 2010;8:5150-8.

19. Qiu JL, Fiil BK, Petersen K, Nielsen HB, Botanga CJ, Thorgrimsen S, et al. Arabidopsis MAP kinase 4 regulates gene expression through transcription factor release in the nucleus. EMBO J. 2008;27:2214-21.

20. Usadel B, Nagel A, Thimm O, Redestig H, Blaesing OE, Palacios-Rojas N, et al. Extension of the visualization tool MapMan to allow statistical analysis of arrays, display of corresponding genes, and comparison with known responses. Plant Physiol. 2005;138:1195-204.

21. Baek D, Pathange P, Chung JS, Jiang J, Gao L, Oikawa A, et al. A stressinducible sulphotransferase sulphonates salicylic acid and confers pathogen resistance in Arabidopsis. Plant Cell Environ. 2010;33(8):1383-92.

22. Gidda SK, Miersch O, Levitin A, Schmidt J, Wasternack C, Varin L. Biochemical and molecular characterization of a hydroxyjasmonate sulfotransferase from Arabidopsis thaliana. J Biol Chem. 2003;278(20):17895-900.

23. Ulm R, Baumann A, Oravecz A, Máté Z, Adám E, Oakeley EJ, et al. Genomewide analysis of gene expression reveals function of the bZIP transcription factor HY5 in the UV-B response of Arabidopsis. Proc Natl Acad Sci U S A. 2004;101(5):1397-402.

24. Hou B, Lim EK, Higgins GS, Bowles DJ. N-glucosylation of cytokinins by glycosyltransferases of Arabidopsis thaliana. J Biol Chem. 2004;279(46):47822-32.

25. Seo YS, Kim EY, Kim WT. The Arabidopsis sn-1-specific mitochondrial acylhydrolase AtDLAH is positively correlated with seed viability. J Exp Bot. 2011;62(15):5683-98.

26. Yang H, Yang S, Li Y, Hua J. The Arabidopsis BAP1 and BAP2 genes are general inhibitors of programmed cell death. Plant Physiol. 2007;145(1):135-46.

27. Kusano M, Tohge T, Fukushima A, Kobayashi M, Hayashi N, Otsuki H, et al. Metabolomics reveals comprehensive reprogramming involving two 
independent metabolic responses of Arabidopsis to UV-B light. Plant J. 2011;67(2):354-69.

28. Ali A, Cheol Park H, Aman R, Ali Z, Yun DJ. Role of HKT1 in Thellungiella salsuginea, a model extremophile plant. Plant Signal Behav. 2013;8:8.

29. Ellouzi H, Ben Hamed K, Hernández I, Cela J, Müller M, Magné C, et al. A comparative study of the early osmotic, ionic, redox and hormonal signaling response in leaves and roots of two halophytes and a glycophyte to salinity. Planta. 2014;240(6):1299-317.

30. Cheng MC, Liao PM, Kuo WW, Lin TP. The Arabidopsis ethylene response factor 1 regulates abiotic stress-responsive gene expression by binding to different cis-acting elements in response to different stress signals. Plant Physiol. 2013;162(3):1566-82.

31. Taji T, Seki M, Satou M, Sakurai T, Kobayashi M, Ishiyama K, et al. Comparative genomics in salt tolerance between Arabidopsis and Arabidopsis-related halophyte salt cress using Arabidopsis microarray. Plant Physiol. 2004;135(3):1697-709

32. Wong CE, Li Y, Labbe A, Guevara D, Nuin P, Whitty B, et al. Transcriptional profiling implicates novel interactions between abiotic stress and hormonal responses in Thellungiella, a close relative of Arabidopsis. Plant Physiol. 2006;140(4):1437-50.

33. Kaveh R, Li YS, Ranjbar S, Tehrani R, Brueck CL, Van Aken B. Changes in Arabidopsis thaliana gene expression in response to silver nanoparticles and silver ions. Environ Sci Technol. 2013:47(18):10637-44.

34. Sham A, Al-Azzawi A, Al-Ameri S, Al-Mahmoud B, Awwad F, Al-Rawashdeh $A$, et al. Transcriptome analysis reveals genes commonly induced by botrytis Cinerea infection, cold, drought and oxidative stresses in Arabidopsis. PLoS One. 2014:9(11):e113718.

35. Rauhut T, Luberacki B, Seitz HU, Glawischnig E. Inducible expression of a Nep1like protein serves as a model trigger system of camalexin biosynthesis. Phytochemistry. 2009;70:185-9.

36. Zhao J, Last RL. Coordinate regulation of the tryptophan biosynthetic pathway and indolic phytoalexin accumulation in Arabidopsis. Plant Cell. 1996;8(12):2235-44.

37. Yin R, Frey M, Gierl A, Glawischnig E. Plants contain two distinct classes of functional tryptophan synthase beta proteins. Phytochemistry. 2010;71:1667-72

38. Sønderby IE, Geu-Flores F, Halkier BA. Biosynthesis of glucosinolates-gene discovery and beyond. Trends Plant Sci. 2010;15:283-90.

39. Wittstock U, Halkier BA. Cytochrome P450 CYP79A2 from Arabidopsis thaliana $\mathrm{L}$. Catalyzes the conversion of $\mathrm{L}$-phenylalanine to phenylacetaldoxime in the biosynthesis of benzylglucosinolate. J Biol Chem. 2000;275(19):14659-66.

40. Gigolashvili T, Berger B, Mock HP, Müller C, Weisshaar B, Flügge UI. The transcription factor HIG1/MYB51 regulates indolic glucosinolate biosynthesis in Arabidopsis thaliana. Plant J. 2007:50(5):886-901.

41. Kilian J, Whitehead D, Horak J, Wanke D, Weinl S, Batistic O, et al. The AtGenExpress global stress expression data set: protocols, evaluation and model data analysis of UV-B light, drought and cold stress responses. Plant J. 2007;50(2):347-63.

42. Pfalz M, Mikkelsen MD, Bednarek P, Olsen CE, Halkier BA, Kroymann J. Metabolic engineering in Nicotiana benthamiana reveals key enzyme functions in Arabidopsis indole glucosinolate modification. Plant Cell. 2011;23(2):716-29.

43. Bednarek P, Pislewska-Bednarek M, Svatos A, Schneider B, Doubsky Mansurova $\mathrm{M}$, et al. A glucosinolate metabolism pathway in living plant cells mediates broad-spectrum antifungal defense. Science. 2009;323:101-6.

44. Clay NK, Adio AM, Denoux C, Jander G, Ausubel FM. Glucosinolate metabolites required for an Arabidopsis innate immune response. Science. 2009;323:95-101.

45. Piotrowski M, Schemenewitz A, Lopukhina A, Müller A, Janowitz T, Weiler EW, et al. Desulfoglucosinolate sulfotransferases from Arabidopsis thaliana catalyze the final step in the biosynthesis of the glucosinolate core structure. J Biol Chem. 2014;279(49):50717-25.

46. Klein M, Reichelt M, Gershenzon J, Papenbrock J. The three desulfoglucosinolate sulfotransferase proteins in Arabidopsis have different substrate specificities and are differentially expressed. FEBS J. 2006;273(1):122-36.

47. Schön M, Töller A, Diezel C, Roth C, Westphal L, Wiermer M, et al. Analyses of wrky 18 wrky 40 plants reveal critical roles of SA/EDS1 signaling and indole-glucosinolate biosynthesis for Golovinomyces orontii resistance and a loss-of resistance towards Pseudomonas syringae pv. tomato AvrRPS4. Mol Plant Microbe Interact. 2013;26(7):758-67.
48. Robatzek S, Somssich IE. Targets of AtWRKY6 regulation during plant senescence and pathogen defense. Genes Dev. 2002;16(9):1139-49.

49. Devaiah BN, Karthikeyan AS, Raghothama KG. WRKY75 transcription factor is a modulator of phosphate acquisition and root development in Arabidopsis. Plant Physiol. 2007;143(4):1789-801.

50. Encinas-Villarejo S, Maldonado AM, Amil-Ruiz F, de los Santos B, Romero F, Pliego-Alfaro $\mathrm{F}$, et al. Evidence for a positive regulatory role of strawberry (Fragaria $\mathrm{x}$ ananassa) Fa WRKY1 and Arabidopsis At WRKY75 proteins in resistance. J Exp Bot. 2009;60(11):3043-65.

51. Li Z, Peng J, Wen X, Guo H. Gene network analysis and functional studies of senescence-associated genes reveal novel regulators of Arabidopsis leaf senescence. J Integr Plant Biol. 2012;54(8):526-39.

52. Gao QM, Venugopal S, Navarre D, Kachroo A. Low oleic acid-derived repression of jasmonic acid-inducible defense responses requires the WRKY50 and WRKY51 proteins. Plant Physiol. 2011;155(1):464-76.

53. Wan J, Zhang S, Stacey G. Activation of a mitogen-activated protein kinase pathway in Arabidopsis by chitin. Mol Plant Pathol. 2004;5(2):125-35.

54. Zheng Z, Qamar SA, Chen Z, Mengiste T. Arabidopsis WRKY33 transcription factor is required for resistance to necrotrophic fungal pathogens. Plant $\mathrm{J}$. 2006;48(4):592-605.

55. Lippok B, Birkenbihl RP, Rivory G, Brümmer J, Schmelzer E, Logemann E, et al. Expression of AtWRKY33 encoding a pathogen- or PAMP-responsive WRKY transcription factor is regulated by a composite DNA motif containing $\mathrm{W}$ box elements. Mol Plant Microbe Interact. 2007:20(4):420-9.

56. Birkenbihl RP, Diezel C, Somssich IE. Arabidopsis WRKY33 is a key transcriptional regulator of hormonal and metabolic responses toward Botrytis cinerea infection. Plant Physiol. 2012;159(1):266-85.

57. Schuhegger R, Rauhut $T$, Glawischnig E. Regulatory variability of camalexin biosynthesis. J Plant Physiol. 2007;164(5):636-44.

58. Vandesompele J, De Preter K, Pattyn F, Poppe B, Van Roy N, De Paepe A, et al. Accurate normalization of real-time quantitative RT-PCR data by geometric averaging of multiple internal control genes. Genome Biol. 2002;3(7):research0034-0034.11.

59. LGC Genomics GmbH. [www.lgcgenomics.com]

60. "Consensus Assessment of Sequence And VAriation", Illumina's sequencing analysis software. [support.illumina.com/downloads/casava_182.html]

61. Babraham Bioinformatics - FastQC. [www.bioinformatics.babraham.ac.uk projects/fastqc/]

62. CLC Genomics Workbench. [www.clcbio.com/products/clc-genomicsworkbench/]

63. Benjamini $Y$, Hochberg $Y$. Controlling the false discovery rate - a practical and powerful approach to multiple testing. J Royal Stat Soc B. 1995;57:289-300.

64. OakLabs GmbH. [www.oak-labs.com]

65. The Arabidopsis Information Resource (TAIR). [www.arabidopsis.org]

66. Nour-Eldin HH, Hansen BG, Norholm MH, Jensen JK, Halkier BA. Advancing uracil-excision based cloning towards an ideal technique for cloning PCR fragments. Nucleic Acids Res. 2006;34(18), e122.

67. Schuhegger R, Nafisi M, Mansourova M, Petersen BL, Olsen CE, Svatos A, et al. CYP71B15 (PAD3) catalyzes the final step in camalexin biosynthesis. Plant Physiol. 2006;141:1248-54.

\section{Submit your next manuscript to BioMed Central and take full advantage of:}

- Convenient online submission

- Thorough peer review

- No space constraints or color figure charges

- Immediate publication on acceptance

- Inclusion in PubMed, CAS, Scopus and Google Scholar

- Research which is freely available for redistribution 